\title{
THE PHYSICS OF MOLECULAR SHOCKS IN YSO OUTFLOWS
}

\author{
DAVID HOLLENBACH \\ MS 245-3, NASA Ames Research Center \\ Moffett Field, CA, 94035-1000, USA
}

\begin{abstract}
Shock waves light up the jets, winds and outflows around YSOs and diagnose the physical conditions and processes resident in these regions. This paper discusses the differences between the jet/wind shock and the ambient shock, between $\mathrm{C}$ shocks and $\mathrm{J}$ shocks, and between the shocks produced by pure jets and by collimated wide angle winds. Basic shock physics is briefly reviewed, with a special focus on the temperature structure in shocks and the Wardle instability of $\mathrm{C}$ shocks. Application is made to the origin of shocked $\mathrm{H}_{2}$ emission and to $\mathrm{H}_{2} \mathrm{O}$ masers.
\end{abstract}

\section{Introduction}

Protostellar birth is marked by the powerful ejection of a significant fraction of the accreting material by the rotating, magnetized, luminous protostar. These outflows or protostellar winds are extremely supersonic; typically, their speeds $\left(\gtrsim 100 \mathrm{~km} \mathrm{~s}^{-1}\right.$ ) are of order the escape speed from the stellar surface or from the inner disk, whereas the internal sound speed or the sound speed in the ambient medium surrounding the protostar is $\lesssim 10 \mathrm{~km}$ $\mathrm{s}^{-1}$. Therefore, strong shocks form both in the wind and where the wind impacts the circumstellar material.

A working description of a shock wave is a region where a supersonic, coherent flow of gas is suddenly decelerated, heated and compressed. From the reference frame of the shock wave, the ordered supersonic flow of particles into the shock is suddenly largely randomized, leading to an irreversible increase of entropy and a rise in temperature. This process is often collisional and may be visualized as a flow of gas particles striking a "wall" of relatively stationary particles, thereby randomizing the directions of their motion. At high shock speeds $\left(\gtrsim 100 \mathrm{~km} \mathrm{~s}^{-1}\right.$ ) the increase in entropy may 


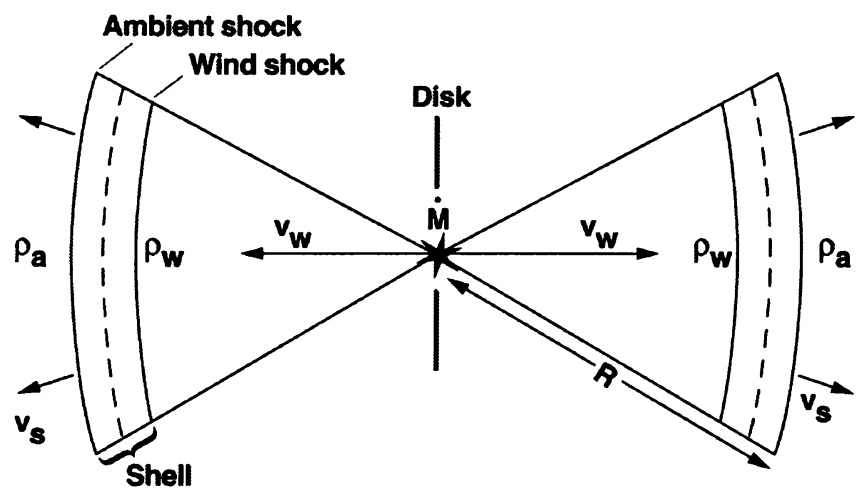

Figure 1. The "two-shock" structure characteristic of winds or jets driving outflows

be produced by collisionless processes, such as the generation and dissipation of plasma turbulence (see references in Draine \& McKee 1993).

Figure 1 schematically illustrates a steady, diverging bipolar flow emanating from near a protostar and producing two shocks as it drives a shell into the ambient medium. The inner shock, sometimes called the "wind" or "jet" shock, decelerates the wind/jet to the shell speed. The outer (downwind) shock, or "ambient" shock, accelerates ambient medium to the shell speed. If the wind is sufficiently collimated to be called a "jet", the jet shock is sometimes called the "Mach disk" and the whole structure including the shell and the two shocks is called the "working surface."

Winds and jets from Young Stellar Objects (YSOs) are sufficiently complex to create shocks in more diverse ways than illustrated in Figure 1. For example, their collimation by magnetic fields and the ambient medium may lead to converging flows towards the polar axis, creating internal shocks. Shocked jet gas can also expand sideways, perpendicular to the jet, leading to a second shock as it encounters ambient medium (see §3.2). In addition, the jets and winds are time dependent, so that the jet/wind or shells may supersonically overtake and shock previously ejected wind or shells. Nevertheless, we shall use the simple case illustrated in Figure 1 to help us understand the basic physics of shocks and the nature of the observed shocks in outflow regions.

There are a number of motivations for studying shocks around YSOs. Shock waves compress, heat and accelerate the preshock medium. The compression forms clumps and may trigger local star formation. On the other hand, the acceleration increases turbulent energy in the ambient medium which may lead to the support or even dispersal of gravitationally bound clouds, and the suppression of more global star formation. The heating al- 
ters the chemistry and dissipates the total kinetic energy of the system. The heating and compression may lead to $\mathrm{H}_{2} \mathrm{O}$ masers, as we will discuss in §3.3. Perhaps more importantly, the heating leads to characteristic shock spectra (often dominated by infrared emission for molecular shocks) which diagnose the presence of activity, the source of the activity, the nature of the outflow, and the characteristics of the ambient preshock medium. For example, the shocks diagnose the wind/jet origin, morphology, mass-loss rate, velocity and mechanical luminosity. They further diagnose the mass, velocity and pressure of swept-up outflow material, and the density structure and chemical abundances in the preshock gas.

A number of papers in this volume have described and reviewed observations of shocks in YSO outflows. Eislöffel, and Noriega-Crespo discuss the $\mathrm{H}_{2} 2 \mu \mathrm{m}$ vibrational emission which delineates shocks in jets and outflows around low mass YSOs. Claussen et al present recent work on $\mathrm{H}_{2} \mathrm{O}$ masers near low mass protostars, and Bachiller \& Gutiérrez reviews shock chemistry. Similarly, shocks have been observed in the outflows around young high mass stars (e.g., the spectacular shocks around $\theta^{1} \mathrm{C}$ in Orion, Allen \& Burton 1993). Bachiller (1996) presents a comprehensive recent review of molecular outflows. Theoretical studies of molecular shocks predict signatures including rotational transitions of $\mathrm{H}_{2}, \mathrm{CO}, \mathrm{OH}$, and $\mathrm{H}_{2} \mathrm{O}$, fine structure emission from [OI], [SI], [SiII], and [FeII], and the chemical enhancement of a number of molecular species (e.g., ; Draine et al 1983; Draine \& Roberge 1984; Flower et al 1985, 1989; Pineau des Forêts et al 1986a,b, 1987, 1989; Hollenbach et al 1989; Hollenbach \& McKee 1989; Neufeld \& Dalgarno 1989a,b; recent review of Draine \& McKee 1993).

\section{Molecular Shock Physics}

In this brief review we shall not discuss the atomic physics of the chemistry or cooling in molecular shock waves (see, e.g., Hollenbach \& McKee 1979, McKee \& Hollenbach 1980, Draine \& McKee 1993), but shall concentrate on shock structure ( $\$ 2.1)$, a magnetic instability in C shocks $(\S 2.2)$, and the application of molecular shock models to outflows (§3.1-3.2) and $\mathrm{H}_{2} \mathrm{O}$ masers (§3.3). However, to understand shock waves one should keep in mind a few facts about molecular shock chemistry and cooling. Above about 300 $\mathrm{K}$, atomic oxygen $\mathrm{O}$ reacts with $\mathrm{H}_{2}$ to form $\mathrm{OH}$ which reacts with $\mathrm{H}_{2}$ to form $\mathrm{H}_{2} \mathrm{O}$. This reaction tends to drive all oxygen not incorporated in $\mathrm{CO}$ into $\mathrm{H}_{2} \mathrm{O}$ in hot postshock gas with high $\mathrm{H}_{2}$ abundances. Further, in postshock gas which is largely molecular, shock cooling is dominated by $\mathrm{H}_{2}$ and $\mathrm{CO}$ at low density $\left(\lesssim 10^{5}-10^{6} \mathrm{~cm}^{-3}\right)$ and by $\mathrm{H}_{2} \mathrm{O}$ and gas-grain collisions at higher density (Kaufman \& Neufeld 1995). 

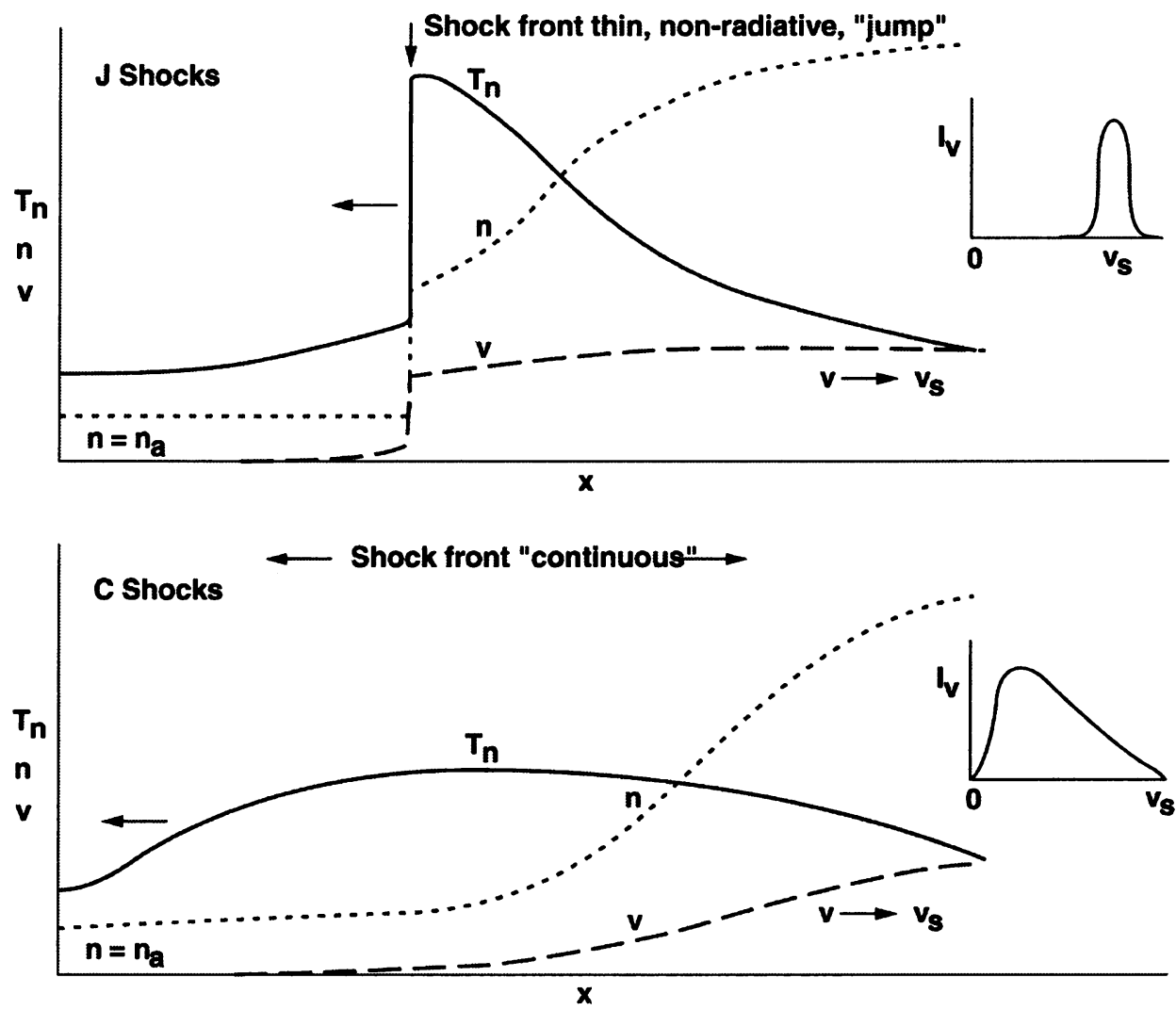

Figure 2. The structure of $\mathrm{J}$ shocks and $\mathrm{C}$ shocks

\subsection{SHOCK STRUCTURE: J SHOCKS AND C SHOCKS}

Figure 2 shows the shock structure and a schematic of the line profile for two types of shock waves: J shocks and C shocks. If we define the "shock front" as the region where the bulk flow is transformed into the random thermal motion of the particles (i.e., where the heat is deposited), then a $\mathbf{J}$ shock can be described as a shock where the heat deposition length is short compared to the cooling length. The shock front is then non-radiative, and one can use the three Rankine-Hugoniot Jump (hence "J") conditions to determine the conditions immediately behind (postshock or "ps") the shock front, which is treated as being infinitely thin.

$$
\begin{gathered}
n_{p s}=4 n_{a}, \\
v_{p s}=(1 / 4) v_{s}, \\
T_{p s}=3.18 \times 10^{5} v_{s 7}^{2} x_{t s}^{-1} \mathrm{~K},
\end{gathered}
$$


where $v$ is measured in the frame of the shock front, $v_{s}$ is the shock velocity (flow velocity into shock front), $v_{s 7}=v_{s} / 100 \mathrm{~km} \mathrm{~s}^{-1}, n$ is the hydrogen nucleus density, $n_{a}$ is the preshock ambient density, and $x_{t s}$ is the number of gas particles per hydrogen nucleus. Note that in Figure 2 we have plotted $v$ in the frame of the ambient gas - often the observer's frame. The emission from the region behind the shock front may be calculated by modeling the subsequent cooling and compression of the postshock gas. In the ambient frame all of the emission occurs between velocities of $(3 / 4) v_{s}$ and $v_{s}$. Therefore, an observer in the ambient frame of an approaching or receding shock would see a relatively narrow line shifted by roughly $v_{s}$ from line center.

Magnetic fields can limit the postshock compression of the cooling gas. If we parameterize the preshock magnetic field component perpendicular to $v_{s}$ as

$$
B_{0 \perp}=b n_{a}^{1 / 2} \mu \mathrm{G}
$$

where $n_{a}$ is in $\mathrm{cm}^{-3}$, then the maximum postshock density is given

$$
n_{m}=77 n_{a} v_{s 7} b^{-1} \text {. }
$$

Note that the Alfvén speed is $v_{A}=1.8 b \mathrm{~km} \mathrm{~s}^{-1}$, and that $b \sim 0.1-3$ under typical interstellar conditions. For shocks which radiate most of the shocked flow energy, the total intensity in all of the cooling radiation is given

$$
I_{T}=9 \times 10^{-5} n_{a} v_{s 7}^{3} \mathrm{erg} \mathrm{cm}^{-2} \mathrm{~s}^{-1} \mathrm{sr}^{-1} .
$$

Equations (5 and 6), which follow from general conservation equations, are valid for both $\mathrm{J}$ and $\mathrm{C}$ shocks. In $\mathrm{J}$ shocks we treat the gas as a single fluid, so that the ions and grains are assumed to be completely frozen to the neutral component. As we shall discuss further below, dissociative shocks $\left(v_{s} \gtrsim 30-50 \mathrm{~km} \mathrm{~s}^{-1}\right)$ are $\mathrm{J}$ shocks, as are lower velocity shocks incident upon interstellar gas with relatively high levels of ionization $\left(x_{i a}>>10^{-6}\right)$.

On the other hand, a different type of shock, the C shock, occurs at relatively low velocities and moderate to low ionizations and depends upon the presence of a magnetic field (Mullan 1971, Draine 1980). When the cold gas contains a well coupled magnetic field, disturbances propagate at the bulk Alfvén speed

$$
v_{A}=\left(\frac{B^{2}}{4 \pi \rho}\right)^{1 / 2} .
$$

Shocks are possible if $v_{s}>v_{A}$, otherwise only damped waves exist. In the partially ionized ISM the bulk medium includes ions which respond nearly instantaneously to changes in the magnetic field and neutrals which couple to the ions by collisions. Here we use "ions" in a general sense to include atomic and molecular ions and charged dust grains. Since such collisions 
are infrequent, it is possible to transmit Alfvén disturbances in just the ion plus field components. These disturbances damp as they propagate, but they travel at the ion Alfvén speed, $v_{i A}=\left(\rho / \rho_{i}\right)^{1 / 2} v_{A}\left(\rho_{i}\right.$ is the ion mass density), which can be very large for small ion fraction. In $\mathrm{C}$ shocks $v_{s}<v_{i A}$, and the magnetic field and ion number density vary continuously, moving as a subsonic wave to transmit information faster than the (neutral) shock speed. If the neutrals as well as the ions vary Continuously across the shock, it is a "C" shock (Draine 1980). The oncoming shock sends a message to the upstream gas via the ions and magnetic field, warning of the approach of compressed, heated and accelerated neutral gas. In the upstream gas, the ions begin to compress and accelerate, so that they drift with respect to the neutrals, gradually heating and accelerating the cold gas via collisions. The neutral flow is continuous when the radiation from the shock front is significant, suppressing the increase in the gas temperature. The thickness or column density of the heated $\mathrm{C}$ shock wave is proportional to $\rho_{i}^{-1}$, or the length scale for each hydrogen molecule to be struck by an ion. On the other hand, the ambipolar heating rate is proportional to $\rho_{i}$. Thus, with increasing ionization fraction, smaller columns of hotter gas are produced until a transition to $\mathrm{J}$ shocks occurs. The columns and temperatures of the $\mathrm{C}$ shocked gas also depend on the preshock magnetic field. The neutrals move through ions which have already been compressed, and their compression is limited by the magnetic field or $b$ (see Eq.(4)). Thus, higher magnetic fields lead to lower ion densities and greater columns of cooler shocked neutral gas.

Nearly the entire flux of a $\mathrm{C}$ shock is produced as infrared emission. In the $\mathrm{C}$ shock the radiation is emitted as the gas is being heated in the shock front; in the $\mathrm{J}$ shock it is given off after the impulsive heating event. To predict the emission from a $\mathrm{C}$ shock we must compute the heating and cooling rates throughout the flow, and we must calculate the continuous structure of the shock front.

These $\mathrm{C}$ shocks are generally non-dissociative (in order that molecular cooling is available), depend on low ionization fractions (so that the ambipolar heating does not drive the molecules to dissociate or collisionally ionize), and form in shocks of relatively low velocity. C shocks occur for $v_{s} \lesssim 40-50 \mathrm{~km} \mathrm{~s}^{-1}$ if $x_{i a} \lesssim 10^{-6}$. The peak temperature rarely exceeds $\sim 3000-5000 \mathrm{~K}$ (to avoid molecular dissociation). The neutral gas radiates copiously before significant acceleration or neutral compression occurs. An observer (at rest with respect to the preshock gas) of an approaching or receding $\mathrm{C}$ shock sees lines broadened by the velocity range of emission with a significant contribution from velocities $<<v_{s}$ (see Figure 2).

The temperature structure in $\mathrm{J}$ and $\mathrm{C}$ shocks are shown in Figures 3 and 4 for two illustrative models. Figure 3 shows a $v_{s}=80 \mathrm{~km} \mathrm{~s}^{-1}$ dissociative 


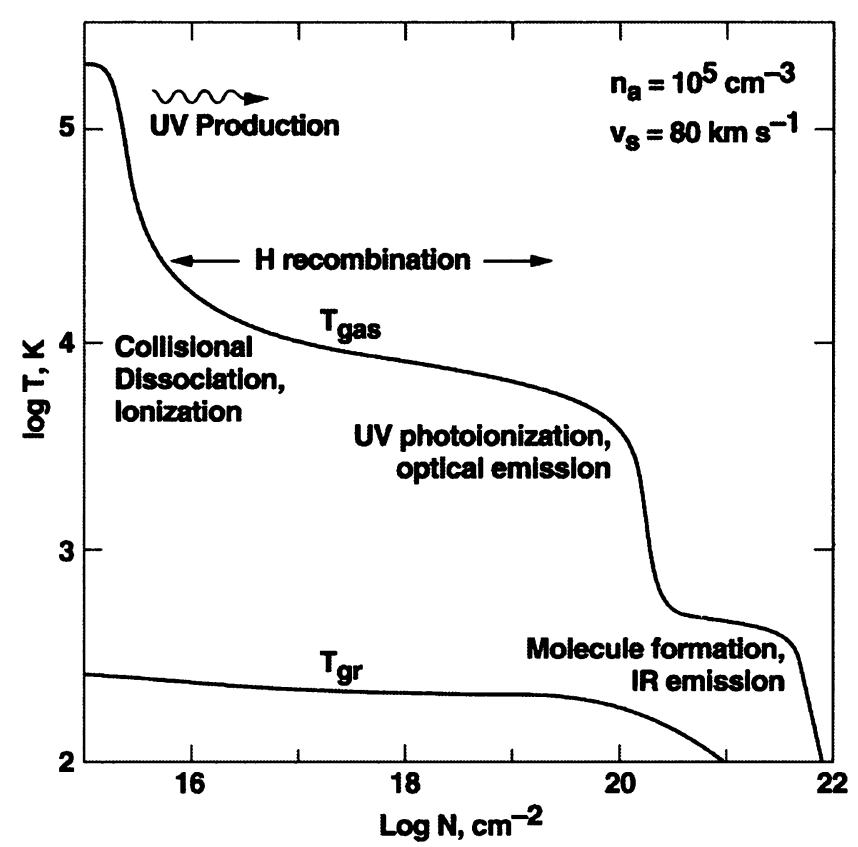

Figure 3. J shock model (Hollenbach \& McKee 1989)

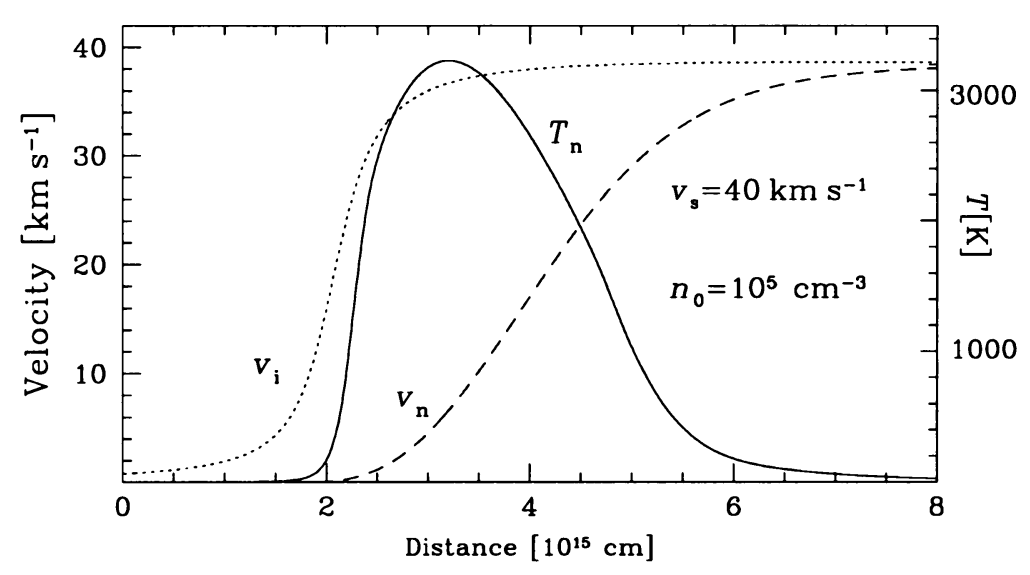

Figure 4. C shock model (Kaufman \& Neufeld 1996a) 
$\mathrm{J}$ shock incident upon ambient gas of density $n_{a}=10^{5} \mathrm{~cm}^{-3}$. Figure 4 shows a $v_{s}=40 \mathrm{~km} \mathrm{~s}^{-1} \mathrm{C}$ shock with the same $n_{a}$. Immediately evident is that the peak gas temperature (see Eq. (3)) in the J shock, where the heat is added impulsively, is much higher than the peak temperature in the $\mathrm{C}$ shock, even when the difference in $v_{s}$ is taken into account. Figure 3 also plots the dust temperature $T_{g r}$; in both $\mathrm{C}$ and $\mathrm{J}$ shocks the dust temperature is much less than the gas temperature because of the weak gas-grain coupling and the efficient radiative cooling of dust.

The $\mathrm{C}$ shock thickness is of order $4 \times 10^{15} \mathrm{~cm}$, whereas the thickness of the $T>100 \mathrm{~K} \mathrm{~J}$-shocked material is $\lesssim 10^{15} \mathrm{~cm}$. The J shock would be even thinner if the heating due to the reformation of molecular hydrogen did not maintain a $\sim 400 \mathrm{~K}$ temperature plateau to $N \sim 4 \times 10^{21} \mathrm{~cm}^{-2}$ (see also Neufeld \& Dalgarno 1989). This effect only occurs for $n_{a} \gtrsim 10^{4}-10^{5} \mathrm{~cm}^{-3}$, so that the newly-formed, vibrationally-excited $\mathrm{H}_{2}$ can be collisionally deexcited before it radiates away its vibrational energy. Thus, $\mathrm{J}$ shocks tend to have smaller columns of hotter (and more atomic) gas than $\mathrm{C}$ shocks.

In the $80 \mathrm{~km} \mathrm{~s}^{-1}$ dissociative $\mathrm{J}$ shock, the gas is first heated to $T \gtrsim 10^{5} \mathrm{~K}$ where it collisionally dissociates and ionizes, and where it emits considerable ultraviolet radiation. The gas cools to $10^{4} \mathrm{~K}$, where the Lyman continuum radiation maintains a temperature plateau through photoionization heating of $\mathrm{H}$. Once these UV photons are absorbed, at $N \sim 10^{20} \mathrm{~cm}^{-2}$ in this model, hydrogen atoms predominate and the gas cools by atomic fine structure emission, especially [OI] $63 \mu \mathrm{m}$. Hollenbach (1985) shows how the [OI] 63 $\mu \mathrm{m}$ luminosity from a dissociative $\mathrm{J}$ shock is proportional to the mass flux into the shock; thus, [OI] $63 \mu \mathrm{m}$ from wind shocks (Figure 1), which are generally radiative $\mathrm{J}$ shocks for low mass protostars, can measure the wind or jet mass loss rate (Cohen et al 1988, Ceccarelli et al 1997).

Molecular hydrogen does not significantly reform behind dissociative J shocks until $T \lesssim 500 \mathrm{~K}$. Therefore, collisional excitation of the $2 \mu \mathrm{m}$ $\mathrm{H}_{2}$ vibrational lines is insignificant, and only a relatively weak emission from newly-formed $\mathrm{H}_{2}$ is predicted (Hollenbach \& McKee 1989). This weak emission has never been definitively detected. However, the heating due to $\mathrm{H}_{2}$ reformation when $n_{a} \gtrsim 10^{4}-10^{5} \mathrm{~cm}^{-3}$ leads to a relatively large column $N \sim 10^{22} \mathrm{~cm}^{-2}$ of $\sim 400 \mathrm{~K}$ gas which is increasingly molecular downstream. $\mathrm{H}_{2} \mathrm{O}$ is formed in this temperature plateau, and the conditions are ripe for $\mathrm{H}_{2} \mathrm{O}$ maser production when $n_{a} \gtrsim 10^{6} \mathrm{~cm}^{-3}$ (see §3.3). Dissociative J shocks also radiate copiously in rotational $\mathrm{OH}, \mathrm{SiO}$, and $\mathrm{CO}$ lines and in the fine structure lines of [OI], [SiII], [FeII], [NeII], and [SI] (Hollenbach \& McKee 1989, Neufeld \& Dalgarno 1989, Haas et al 1991).

Figure 4 demonstrates that $\mathrm{C}$ shock temperatures are never large enough to dissociate $\mathrm{H}_{2}$; however, large columns of $T \sim 1000-3000 \mathrm{~K} \mathrm{H}_{2}$ can be produced, and $\mathrm{H}_{2} 2 \mu \mathrm{m}$ emission can be very luminous. The hot molecular 


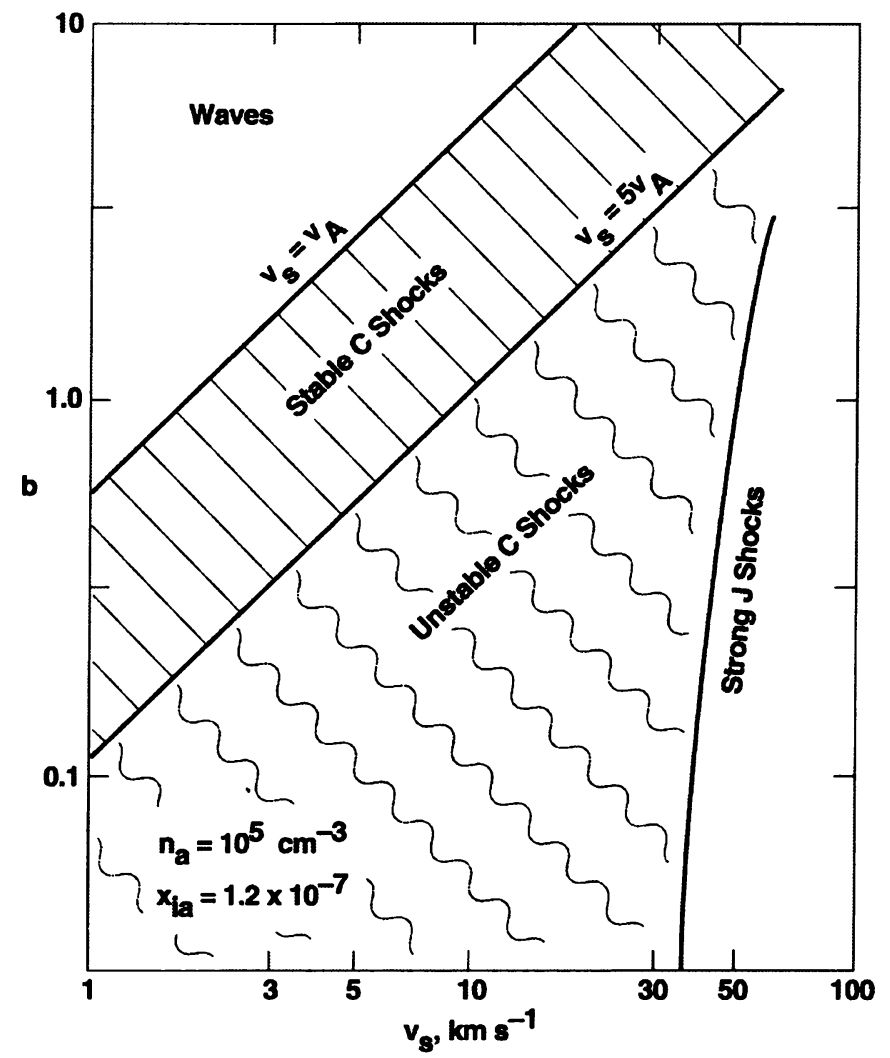

Figure 5. C and J shock parameter space (Hollenbach et al 1989)

gas also rapidly transforms all oxygen not in $\mathrm{CO}$ into $\mathrm{H}_{2} \mathrm{O}$. Hence, intense infrared $\mathrm{H}_{2} \mathrm{O}$ emission is predicted, along with even warmer masers than in J shocks (Neufeld \& Melnick 1991, Melnick et al 1993, Kaufman \& Neufeld 1996b). Figure 4 compares ion and neutral flow velocities measured in the preshock frame, and demonstrates that ions are accelerated first so that they can then drag the neutrals to the shock speed. Pineau des Forêts et al. (this volume) discuss other interesting aspects of $\mathrm{C}$ shocks.

Figure 5 illustrates the parameter space occupied by $\mathrm{C}$ and $\mathrm{J}$ shocks as a function of the shock velocity and preshock magnetic field ( $b$ is defined in Eq. (4)). We have chosen representative values for $n_{a}$ and $x_{i a}$; J shocks become more prevalent if $x_{i a}>>10^{-6}$ (e.g., Hollenbach et al 1989, Smith $\&$ Brand 1990). In the upper left corner $v_{s}>v_{A}$ and no shocks forms. C shocks form between the solid lines. J shocks form when the shock velocities are high enough ( $\gtrsim 40 \mathrm{~km} \mathrm{~s}^{-1}$ ) to dissociate $\mathrm{H}_{2}$, which reduces the cooling and leads to impulsive shock heating. 

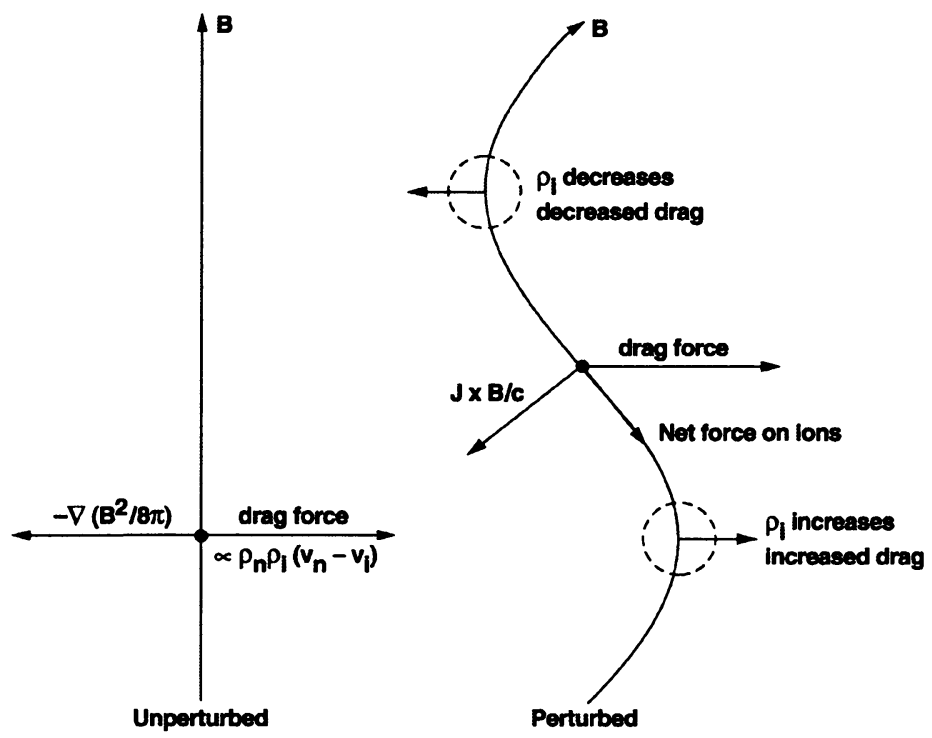

Figure 6. The Wardle Instability (adapted from Draine \& McKee 1993)

\subsection{THE WARDLE INSTABILITY OF C SHOCKS}

Wardle $(1990,1991 \mathrm{a}, \mathrm{b})$ described an important dynamical instability in $\mathrm{C}$ shocks involving deformation of the magnetic field. Figure 6 schematically illustrates the Wardle instability. Consider a plane parallel shock with $B$ perpendicular to $v_{s}$. The ions and ( $B$ field) move ahead of the compressed neutral gas, driven by the $\nabla\left(B^{2} / 8 \pi\right)$ force until a steady state wave forms, retarded by the drag force of the neutrals on the ions. Consider a perturbation of the magnetic field as in Figure 6. The drag force now has a component parallel to the local $B$ field that cannot be balanced by the $\mathbf{J} \times \mathbf{B}$ force, and ions will therefore be accelerated along field lines to collect in magnetic "valleys". As a consequence, $\rho_{i}$ will increase in the valleys, the drag force will increase there, and the field lines will be further distorted. Wardle found $\mathrm{C}$ shocks to be unstable for $M_{A} \gtrsim 5$ (see Figure 5).

This significant discovery left shock modelers in a bad state. Until the non-linear development of the clumps and spectra produced by these $\mathrm{C}$ shocks could be followed by numerical MHD codes, there was no consistent way to compare observations with shock models, since a large portion of $\mathrm{C}$ shock parameter space (Figure 5) was now uncertain. The Chamonix meeting therefore marked an historic development in interstellar shock modeling as two teams (MacLow \& Smith 1997; Stone 1997, Neufeld \& Stone 1997) reported results from $2 \mathrm{D}$ MHD codes which treated the weakly coupled ions and neutrals separately and followed the Wardle instability to saturation. 
The surprising and interesting (though possibly disappointing to the code developers) results reported by Neufeld \& Stone is that the C shock spectra from Wardle unstable shocks do not appreciably deviate from the spectra found by the older $1 \mathrm{D}$ steady state $\mathrm{C}$ shock codes which suppressed the instability. The neutral gas emits before it is appreciably clumped. Thus, the older model spectra (e.g., Draine et al 1983, Draine \& Roberge 1984) are still applicable. MacLow \& Smith emphasize that the weaker, high excitation lines are affected more, and that the instability may lead to measurable spectral variations with time.

\section{Applications}

\subsection{ASPECTS OF THE TWO-SHOCK PICTURE}

A useful approximation to the two-shock model illustrated in Figure 1 is that often a pseudo "steady state" is established where the pressure in the shell is equal to the ram pressure of the supersonic gas hitting it from both sides. Therefore the ram pressure of the ambient gas equals the ram pressure of the overtaking wind

$$
\rho_{a} v_{s}^{2} \simeq \rho_{w}\left(v_{w}-v_{s}\right)^{2} .
$$

If $\rho_{a}>>\rho_{w}$ (a "light" jet/wind), then $v_{s} \simeq\left(\rho_{w} / \rho_{a}\right)^{1 / 2} v_{w}$, the shell moves much slower than the wind. If the wind shock is radiative, the total luminosity of the wind shock is greater than the ambient shock by a factor $\left(v_{w} / v_{s}\right)$. However, the wind shock will likely be a dissociative $\mathrm{J}$ shock radiating much of its luminosity in the $\mathrm{UV}$, and the ambient shock may dominate the $\mathrm{H}_{2}$ emission. The mass in the shell is dominated by the flux of material from the ambient shock by a factor $\left(v_{w} / v_{s}\right)$.

On the other hand, if $\rho_{a}<<\rho_{w}$ (sometimes called a "heavy" jet/wind), then the shell propagates at nearly the wind speed, $v_{s} \sim v_{w}$, and the wind shock is a slow shock while the ambient shock may be a dissociative $\mathrm{J}$ shock. This occurs early in the evolution of a jet/wind, or possibly when the wind breaks out of the core into a low density interclump medium. In this case, the ambient shock dominates the luminosity whereas the mass in the shell is mostly injected by the wind.

We note that Eq.(8) can only be strictly valid when the ambient gas and wind strike the shock fronts perpendicularly. Wilkin (1996, this volume) has found analytic solutions to more general cases, and applied these solutions to bow and outflow shocks near protostars.

Davis and Eislöffel (1995) noted and studied an interesting predicted correlation between the ambient shock luminosity $L_{s a}$ and the mechanical luminosity $L_{C O}$ of the swept material between the two shocks (Figure 1). 
$L_{s a}$ is given

$$
L_{s a}=\frac{1}{2} \rho_{a} v_{s}^{3} A_{s a}
$$

where $A_{s a}$ is the area of the shock. $L_{C O}$ is derived from $\mathrm{CO}$ measurements. The earliest and most prevalent signature of protostellar outflows has been CO rotational line emission detected in line wings, at $v \sim 5-30 \mathrm{~km} \mathrm{~s}^{-1}$ from line center, indicating material that was not gravitationally bound to the star-forming clump and was therefore an "outflow". The CO measurements indicated hydrogen masses $M_{C O} \lesssim 200 \mathrm{M}_{\odot}$; such large masses indicate that this outflow is not the wind from the protostar/disk, but must be swept-up ambient material (e.g., Lada 1985, Fukui et al 1993, Bachiller 1996). $L_{C O}$ is defined

$$
L_{C O}=\frac{\frac{1}{2} M_{C O} v_{s}^{2}}{\tau}
$$

where $\tau=R / v_{s}$, the flow "lifetime". Assuming any ambient density distribution less steep than $r^{-3}$ and that most of the shell mass is swept-up ambient gas and not shocked wind (light wind, $v_{s}<<v_{w}$ ), $M_{C O} \simeq A_{s a} R \rho_{a}$. Substitution into Eq. (10) leads to

$$
L_{C O} \simeq L_{s a}
$$

The current ambient shock luminosity is equal to the shell kinetic energy divided by its age. Davis \& Eislöffel (1995) do not actually observe this correlation in mapping 5 sources, but they attribute the discrepancies to difficulties in determining $v_{s}$ and $\tau$ from CO observations, the extinction at $2 \mu \mathrm{m}$ to correct for the intrinsic $L_{s a}$, and the possibility of a heavy wind.

\subsection{JETS AND WIDE ANGLE WINDS}

Observations of protostellar outflows have demonstrated two types of phenomena whose relationship is not yet clear. On the one hand, optical observations of relatively unobscured regions of the outflow reveal tightly collimated ( $\lesssim 100 \mathrm{AU}$ ) jets which extend to large distances $(\gtrsim 0.1 \mathrm{pc})$ from the protostar (e.g., Mundt \& Fried 1983, Bachiller 1996). These jets are generally observed in $\mathrm{H} \alpha, \operatorname{SII}(6730 \AA)$, and other low excitation optical emission lines, presumably excited in the $T \sim 10^{4} \mathrm{~K}$ ionized gas behind dissociative $\mathrm{J}$ shocks (Figure 3 ) formed either internal to the jet or at the jet/ambient gas interface. On the other hand, the CO outflows, especially those in more obscured regions, generally show a much less collimated structure (references in Bachiller 1996; Cernicharo et al, Padman et al, this volume).

Two classes of theories have arisen to reconcile or unify these seemingly contradictory or disparate phenomena. On the one hand are theories that assume that the basic stellar or disk wind is very tightly collimated into a 


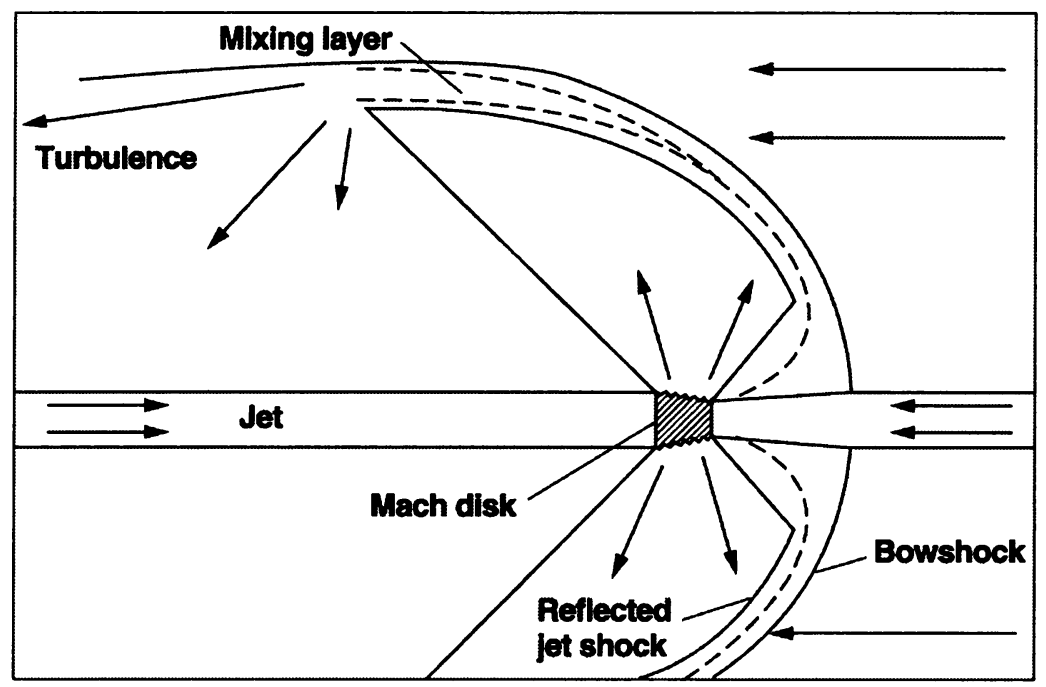

Figure 7. A jet creating a bow shock (adapted from Raga \& Cabrit 1993)

jet by an unspecified mechanism. However, the interaction of this jet with the ambient medium leads to a bow shock and turbulent entrainment of ambient material (the $\mathrm{CO}$ outflow) that extends far from the jet axis (e.g., Raga \& Cabrit 1993, Masson \& Chernin 1993, Stahler 1994, Chernin \& Masson 1995). On the other hand, Shu et al (1995) (see also Shu \& Shang, this volume) have derived the streamlines of the MHD wind driven from the inner accretion disk of a protostar by the interaction with the magnetic dipole of a rotating star. They find that the pinching effect of the toroidal component of the magnetic field set up by this rotating configuration leads to a natural collimation of the wind. The density in the wind is higher along the pole than away from the cylindrical axis, and thus the emission measure of the optical lines strongly peak along the axis, giving the appearance of a jet. However, the streamlines are actually quite radial, and there is still considerable mass loss, $\sim 0.5$ of the total, which is ejected at relatively wide angles to the axis. The wind is really a "collimated wide angle wind." This second class of model, therefore, relies on the direct impact of the wide angle component to drive the less collimated $\mathrm{CO}$ outflows.

Both of these classes of models can be understood by extending the simple two shock picture of Figure 1. Here, we shall only focus on the shocks and the shock physics of these models. Figure 7 shows a schematic figure of one of the first class of models, where a solitary jet sets up a bow shock. In this model the pressurized shell between the jet shock and the ambient shock can "squirt" gas out sideways at high speed. This ejection from the 


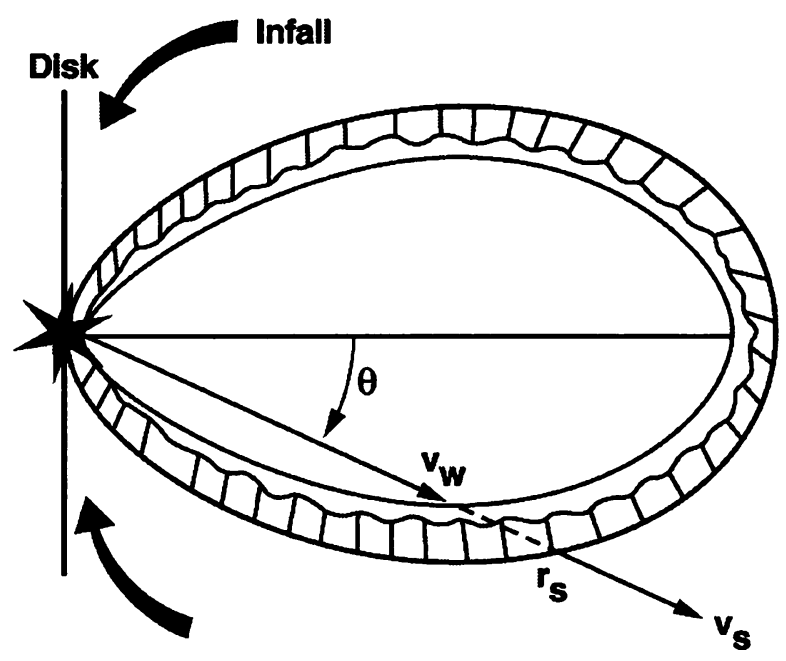

Figure 8. The wide angle collimated wind (adapted from Shu et al 1991)

working surface acts then like a much less collimated wind, which interacts with the ambient gas to form an inner shock of the deflected jet with an outer ambient bow shock (Figure 7). Material coming off the distant reaches of the bow, far from the axis, form vortices and turbulence downstream which extends along the jet towards the source. The bow and turbulence entrain the more extended, less collimated $\mathrm{CO}$ outflow. A variation of this class of models is that the turbulence set up by the shear of the jet through the ambient gas creates a thick turbulent layer, and the $\mathrm{CO}$ outflow is entrained in this turbulent layer (Stahler 1994).

Figure 8 shows a schematic of the collimated wide angle wind model. The shape of the outflow shell depends on the intrinsic collimation of the wind $\rho_{w}(\theta)$ and the density distribution $\rho_{a}(\theta)$. In both Figures 7 and 8 we have only illustrated one lobe of the bipolar flow, and the accretion disk is viewed edge-on and vertical. $\mathrm{Li} \& \mathrm{Shu}(1996)$ show that using the Shu et al (1995) wind solution for $\rho_{w}(\theta)$ and the density contours of flattened magnetized cores for $\rho_{a}(\theta)$, one can produce in the shell the observed mass in the CO outflow as a function of velocity (Masson \& Chernin 1992).

Hartigan et al (1996) present a good review of the various possibilities for the origin of the observed $\mathrm{H}_{2}$ emission in bow shocks. We highlight here a few important observations and suggestions. The $2 \mu \mathrm{m}$ vibrational lines lie $6000-18,000 \mathrm{~K}$ above ground, and the line ratios suggest temperatures $\sim 2000 \mathrm{~K}$. Brand et al $(1988,1989)$ has shown that observations of different regions often show remarkably similar line ratios, suggestive of an effective thermostating mechanism. Smith et al (1991) model $\mathrm{H}_{2}$ spectra from bow 
shocks, and show that the ambient shock gets progressively more oblique further back on the bow. The head of the bow, therefore, has the highest ambient shock velocity which in many cases may be a dissociative $\mathrm{J}$ shock, weak in $\mathrm{H}_{2} 2 \mu \mathrm{m}$ emission. Further back on the bow, the shock velocity (the perpendicular component of the ambient flow velocity striking the bow shell) drops below the critical velocity $v_{c r}$ for the $\mathrm{J} / \mathrm{C}$ transition, and a strong (oblique) $\mathrm{C}$ shock is produced, intense in $\mathrm{H}_{2} 2 \mu \mathrm{m}$ emission. As long as the bow speed $v_{b}$ into the ambient gas exceeds $v_{c r}$, a range of $\mathrm{C}$ shock velocities from the dissociation limit down to very weak shocks are therefore produced. If the beam encompasses the entire bow structure, the resultant $\mathrm{H}_{2}$ spectra are insensitive to $v_{b}$ for $v_{b}>v_{c r}$.

We further note that global bow shocks and shells are created by the jets and collimated winds discussed above, but that much smaller bow shocks may be produced if the ambient gas has small clumps which are overtaken by the shell. In this case, a small bow forms around the clump, with the head of the bow pointing toward the outflow source. The global bow points away. Such clumps might be produced by episodic mass loss, which sends previous generations of global bow shocks through the ambient gas. Wardle instabilities in these shocks produce clumps of size scale the order of the thickness of the $\mathrm{C}$ shocks, which may be of order $10^{16}-10^{17}$ $\mathrm{cm}$ for $n \sim 10^{5}-10^{4} \mathrm{~cm}^{-3}$ (Figure 4).

\section{3. $\mathrm{H}_{2} \mathrm{O}$ MASERS ASSOCIATED WITH YSOS}

Interstellar $\mathrm{H}_{2} \mathrm{O}$ masers at $22 \mathrm{GHz}$ often appear to be individual clumps, streaming away from some center of activity at velocities up to $200 \mathrm{~km} \mathrm{~s}^{-1}$. Individual features have apparent sizes of $10^{13} \mathrm{~cm}$ and brightness temperatures usually in the range $T_{b} \sim 10^{11}-10^{14} \mathrm{~K}$ (Genzel 1986). The isotropic luminosity of individual maser spots range from $\lesssim 10^{-6}$ to $0.08 \mathrm{~L}_{\odot}$ in the Galaxy (Walker et al 1982). Pumping by an external source of radiation is ruled out by observations (e.g., Genzel 1986), and an internal source of pump energy, such as produced in a shock, seems required. The development of powerful shocks in maser regions is inevitable in light of the high velocities observed in the sources, and the $\mathrm{H}_{2} \mathrm{O}$ maser luminosity correlates with the mechanical luminosity in the observed outflows (Felli et al 1992), as would be expected in a shock model.

Litvak (1969), Strelnitski \& Sunyaev (1973), Schmeld et al (1976), Hollenbach et al (1987), Elitzur et al (1989), Kaufman \& Neufeld (1996b), and Hollenbach et al (1997) propose that $\mathrm{H}_{2} \mathrm{O}$ masers originate in the warm molecular gas behind shock waves driven by YSO winds. C shocks simply heat the molecular gas; fast $\mathrm{J}$ shocks dissociate the molecular gas, but as $\mathrm{H}_{2}$ reforms in the postshock gas, it delivers part of its formation energy as heat 


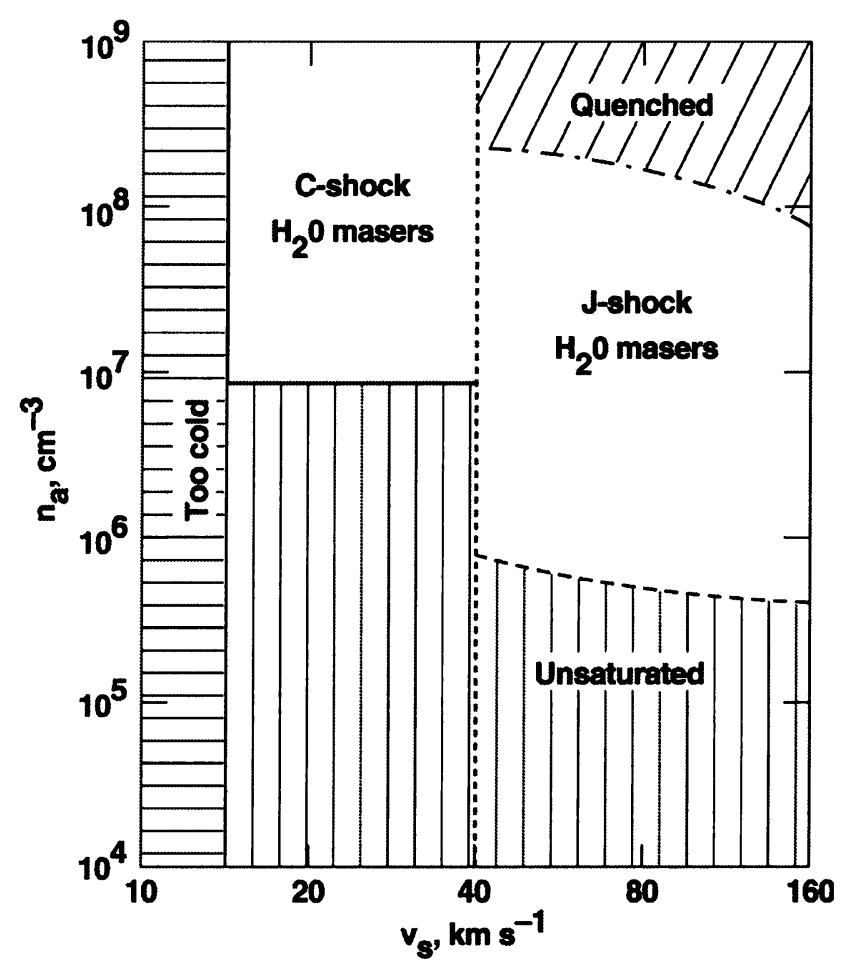

Figure 9. The shock parameter space for strong $22 \mathrm{GHz} \mathrm{H}_{2} \mathrm{O}$ masers, assuming $v_{A} \sim 1$ $\mathrm{km} \mathrm{s}^{-1}$ and $x_{i a} \lesssim 10^{-6}$ (Hollenbach et al 1997)

which maintains a large postshock molecular column at $T \sim 400 \mathrm{~K}$ (Figure $3)$. In either case, the warm $T \gtrsim 300 \mathrm{~K}$ postshock gas drives all oxygen not locked in $\mathrm{CO}$ to form $\mathrm{H}_{2} \mathrm{O}$, and collisionally excites the maser levels which typically lie $\gtrsim 600 \mathrm{~K}$ above the ground state. Shock compression (especially in J shocks) helps the gas attain sufficiently high densities to produce strong masers. The sheet-like geometry of the shocked gas allows infrared photons to escape normal to the sheet, thereby enhancing nonLTE level populations necessary for the maser inversion. It also provides long coherence paths in the plane of the shock for the maser lines.

Figure 9 summarizes the shock parameter space which produces $22 \mathrm{GHz}$ $\mathrm{H}_{2} \mathrm{O}$ masers. The vertical dashed line at $v_{s} \sim 40 \mathrm{~km} \mathrm{~s}^{-1}$ marks the approximate boundary between $\mathrm{C}$ and $\mathrm{J}$ shocks. Above the dash-dot line, the maser is quenched in $\mathrm{J}$ shocks. Below the dashed and solid line, the maser is unsaturated and weak. J shocks in the range $\left(v_{s} \gtrsim 40 \mathrm{~km} \mathrm{~s}^{-1}, 10^{6}\right.$ $\left.\mathrm{cm}^{-3} \lesssim n_{a} \lesssim 10^{8} \mathrm{~cm}^{-3}\right)$ and $\mathrm{C}$ shocks in the range $\left(15 \mathrm{~km} \mathrm{~s}^{-1} \lesssim v_{s} \lesssim 40\right.$ $\mathrm{km} \mathrm{s}^{-1}, 10^{7} \mathrm{~cm}^{-3} \lesssim n_{a} \lesssim 10^{9} \mathrm{~cm}^{-3}$ ) produce strong, saturated, beamed 22 
$\mathrm{GHz} \mathrm{H}_{2} \mathrm{O}$ masers.

Genzel (1986) reviews the observations of $\mathrm{H}_{2} \mathrm{O}$ masers and we summarize here the shock model explanation of the data. Their space velocities are often $\gtrsim 30 \mathrm{~km} \mathrm{~s}^{-1}$; this is the velocity required to produce strong $\mathrm{C}$ or $\mathrm{J}$ shocks. Their densities are inferred to be $\sim 10^{9} \mathrm{~cm}^{-3}$; a J shock compresses gas of density $\sim 10^{7} \mathrm{~cm}^{-3}$ to this density, while a $\mathrm{C}$ shock requires higher preshock densities. Their spot sizes are about $10^{13} \mathrm{~cm}$; the shock thickness and unsaturated core size are of this order in shock models. Their observed isotropic luminosities range from $10^{-7}-10^{-1} \mathrm{~L}_{\odot}$ and their brightness temperatures $T_{b}$ range from $10^{11}-10^{14} \mathrm{~K}$; the shock models in the parameter range shown in Figure 9 produce these numbers with coherence lengths of $10^{14-15} \mathrm{~cm}$. Lower radial velocity masers tend to have higher $T_{b}$; in shocks, lower radial velocites mean that the line of sight is close to the shock plane, resulting in larger coherence lengths and $T_{b}$. The $\mathrm{H}_{2} \mathrm{O}$ maser luminosity correlates with the mechanical luminosity seen the the $\mathrm{CO}$ outflow; the mass loss produces the shocks which, in turn, produce the masers (see also Eq. 11). Millimeter observations, the detection of other $\mathrm{H}_{2} \mathrm{O}$ maser lines (e.g., Melnick et al 1993), and the observation that there are not enough external photons to pump the maser all point to warm $\gtrsim 300 \mathrm{~K}$ gas where collisions pump the maser; $\mathrm{J}$ shocks produce large columns of $\sim 400 \mathrm{~K} \mathrm{H}_{2} \mathrm{O}$ and $\mathrm{C}$ shocks can produce even warmer $\mathrm{H}_{2} \mathrm{O}$. Finally, Fiebig \& Güsten (1989) have observed the Zeeman splitting of the $22 \mathrm{GHz} \mathrm{H}_{2} \mathrm{O}$ maser in $\mathrm{W} 49$ and estimated the component of the $B$ field along the line of sight to be about $100 \mathrm{mG}$; the shock models predict exactly this order of field in the masing region, relatively independent of the preshock field (Elitzur et al 1989).

Acknowledgements: I gratefully acknowledge the support of the NASA Astrophysical Theory Program, which funds the Center for Star Formation Studies, a consortium of researchers from NASA Ames, University of California at Santa Cruz, and University of California at Berkeley.

\section{References}

Allen, D.A., Burton, M.G. 1993, Nature 363, 54

Bachiller, R. 1996, ARAA 34, 111

Brand, P.W.J.L., Toner, M.P., Geballe, T.R., Webster, A.S. 1989, MNRAS 237, 1009

Brand, P.W.J.L., Moorhouse, A., Burton, M.G., Geballe, T.R., Bird, M., Wade, R. 1988, ApJ Lett. 334, L103

Ceccarelli, C., Haas, M.R., Hollenbach, D.J., Rudolph, A.L. 1997, ApJ 476, 771

Chernin, L., Masson, C. 1995, ApJ 443, 181

Cohen, M., Hollenbach, D.J., Haas, M.R., Erickson, E.F. 1988, ApJ 329, 863

Davis, C.J., Eislöffel, J. 1995, A\&A 300, 851

Draine, B.T. 1980, ApJ 241, 1021 (erratum: 246, 1045)

Draine, B.T., McKee, C.F. 1993, ARAA 31, 373

Draine, B.T., Roberge, W.G. 1984, ApJ 282, 491

Draine, B.T., Roberge, W.G., Dalgarno, A. 1983, ApJ 264, 485 
Elitzur, M., Hollenbach, D.J., McKee, C.F. 1989, ApJ 346, 983

Felli, M., Palagi, F., Tofani, G. 1992, A\&A 255, 293

Fiebig, D., Güsten, R. 1989, A\&A Lett. 214, 333

Flower, D.R., Pineau des Forêts, G., Hartquist, T.W. 1985, MNRAS 216, 775

Flower, D.R., Heck, L., Pineau des Forêts, G. 1989, MNRAS 239, 741

Fukui, Y., Iwata, T., Mizuno, A., Ball, J., Lane, A.P. 1993, in Protostars and Planets III, ed E.H. Levy, J.I. Lunine (Tucson: Univ. Ariz. Press), p603

Genzel, R. 1986, in Masers, Molecules and Mass Outflows in Star Forming Regions, ed. A.D. Haschick (Westform, MA: Haystack Observatory), p233.

Haas, M.R., Hollenbach, D.J., Erickson, E.F. 1991, ApJ 374, 555

Hartigan, P., Carpenter, J.M., Dougados, C., Skrutskie, M.F. 1996, AJ 111, 1278

Hollenbach, D.J. 1985, Icarus 61, 40

Hollenbach, D.J., Chernoff, D., McKee, C.F. 1989, in Infrared Spectroscopy in Astronomy, ed. B. Kaldeich, ESA SP-290, p245

Hollenbach, D.J., Elitzur, M., McKee, C.F. 1997, ApJ, in preparation

Hollenbach, D.J, McKee, C.F. 1979, ApJ Suppl. 41, 555

Hollenbach, D.J., McKee, C.F. 1989, ApJ 342, 306

Hollenbach, D.J., McKee, C.F., Chernoff, D. 1978, in it Star Forming Regions, ed. M. Peimbert \& J. Jugaku (Dordrecht: Reidel), p334

Kaufman, M.J., Neufeld, D.A. 1995, ApJ 418, 263

Kaufman, M.J., Neufeld, D.A. 1996a, ApJ 456, 611

Kaufman, M.J., Neufeld, D.A. 1996b, ApJ 456, 250

Lada, C. 1985, ARAA 23, 267

Litvak, M.M. 1969, Science 165, 855

MacLow, M.M., Smith, M.D. 1997, ApJ, submitted

Masson, C.R., Chernin, L.M. 1992, ApJ Lett. 387, L47

Masson, C.R., Chernin, L.M. 1993, ApJ 414, 230

McKee, C.F., Hollenbach, D.J. 1980, ARAA 18, 219

Melnick, G.J., Menton, K.M., Phillips, T.G., Hunter, T. 1993, ApJ 416, L37

Mullan, D.J. 1971, MNRAS 153, 145

Mundt, R., Fried, J.W. 1983, ApJ Lett. 274, L83

Neufeld, D.A., Dalgarno, A. 1989a, ApJ 340, 869

Neufeld, D.A., Dalgarno, A. 1989b, ApJ 344, 251

Neufeld, D.A., Melnick, G.J. 1991, ApJ 368, 215

Neufeld, D.A., Stone, J.M. 1997, ApJ, submitted

Pineau des Forêts, G., Flower, D., Hartquist, T., Dalgarno, A. 1986a, MNRAS 220, 801

Pineau des Forêts, G., Flower, D., Hartquist, T., Millar, T. 1987, MNRAS 227, 993

Pineau des Forêts, G., Roueff, E., Flower, D.R. 1986b, MNRAS 223, 743

Pineau des Forêts, G., Roueff, E., Flower, D.R. 1989, JChemSocFaradayTrans 85, 1665

Raga, A., Cabrit, S. 1993, A\&A 278, 267

Schmeld, I.K., Strelnitski, V.S., Muzylev, V.V. 1976, SovAstron 20, 4111

Shu, F.H., Najita, J., Ostriker, E., Shang, S. 1995, ApJ Lett. 455, L155

Smith, M.D., Brand, P.W.J.L. 1990, MNRAS 242, 495

Smith, M.D., Brand, P.W.J.L., Moorhouse, A. 1991, MNRAS 248, 451

Stahler, S. 1994, ApJ 422, 616

Stone, J.M 1997, ApJ, submitted

Strelnitski, V.S., Sunyaev, R.A. 1973, SovAstron 16, 579

Walker, R.C., Matsakis, D.N., Garcia-Barreto, J.A. 1982, ApJ 255, 128

Wardle, M. 1990, MNRAS 246, 98

Wardle, M. 1991a, MNRAS 250, 523

Wardle, M. 1991b, MNRAS 251, 119

Wilkin, F.P. 1996, ApJ Lett. 459, L31 